\title{
The Origins of Variability in The Responses of The Glutamatergic Synapse
}

\author{
Vito Di Maio* \\ Institute of Applied Science and Intelligent Systems (ISASI) of CNR, Italy \\ *Corresponding author: Vito Di Maio, Institute of Applied Science and Intelligent Systems (ISASI) of CNR, Italy, Email: vito.dimaio@ \\ cnrit
}

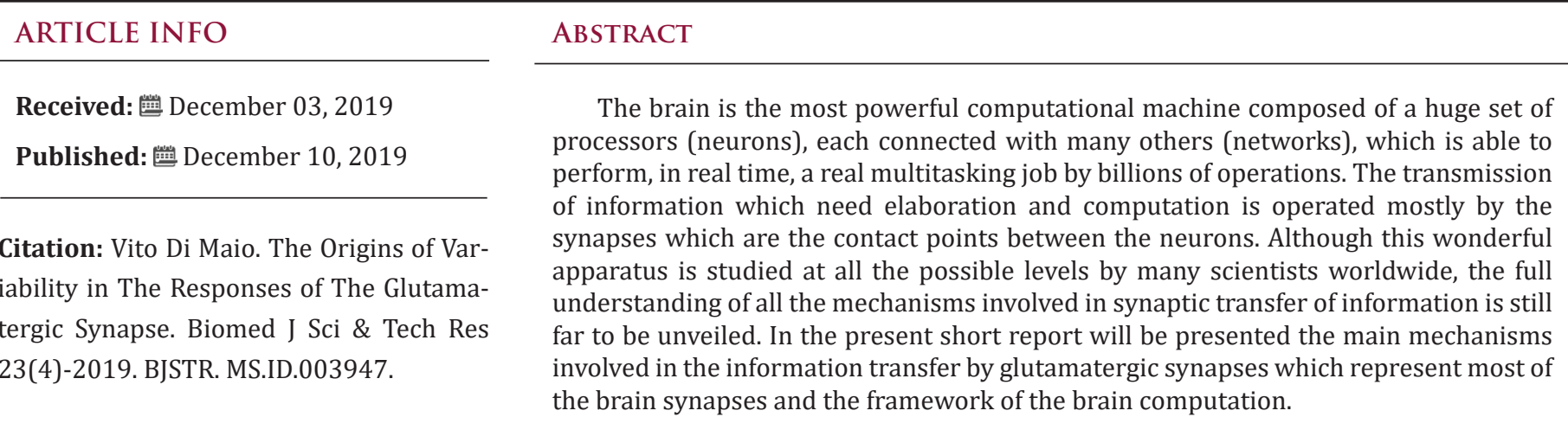

\section{Introduction}

The functional building blocks of the brain are the neurons forming specialized neural networks which interact each other and with the body periphery by the means of specialized loci where information is transferred: the synapses. Glutamatergic synapses represent $80-90 \%$ of the total brain synapses and each single neuron of the cortex and of a subfield of the hippocampal formation receives $3 \times 10^{3}-4 \times 10^{4}$ glutamatergic synaptic contacts [1-2]. Not surprisingly then, the malfunctioning of these synapses is involved in severe brain impairments related to cognitive processes like Parkinson's disease [3], Alzheimer's Disease [4], autism [5] and many others.

Glutamatergic synapses are not simply junctional points transmitting signals which encode information. They act as filters of the information carried by presynaptic neurons performing a first important step of the neuronal computation and elaboration of the information. Although the mechanism of synaptic transmission is simple in principle, it is finely regulated at different levels and the information content manipulated by important mechanisms of control. A part of these mechanisms operates at the presynaptic side, others involve the postsynaptic one, but the activity of other synapse located in the proximity of a given synapse play a significant role too [6]. The main mechanisms are shown in the scheme of
Figure 1. In the following sections the pre, post and extrasynaptic mechanisms influencing the information transfer will be briefly exposed. To this goal we need to have on focus the glutamatergic synaptic structure and how the information flow from the pre to the postsynaptic neuron. Next section and Figure 1 are devoted to this goal.

\section{The Synaptic Structure and Its Functionality}

The synaptic structure plays itself a role in the formation of the synaptic code and of the postsynaptic neural code. Glutamatergic synapses are localized on the top of spines protruding from the shafts of the dendritic tree. A spine is formed by a neck and the head on the top of which there is the Post Synaptic Density (PSD) where the ionotropic glutamatergic receptors (AMPA and NMDA) are colocalized. The biophysical characteristic of the spine forms a specialized, separate, electrical circuit which can vary among synapses and among different neurons producing remarkable effects on the postsynaptic response [6-14]. On the top of the spine's head, at a distance of almost $20 \mathrm{~nm}$ (synaptic cleft), behind the presynaptic membrane, a set of vesicles filled of the neurotransmitter glutamate, are docked to the membrane and, some of them (primed), are ready to be released. 
The functionality of the glutamatergic synapse is apparently simple. In short, the arrival of a presynaptic spike causes the formation of a fusion pore between the membrane of a vesicle and that of the presynaptic surface. The pore quickly expands according to a radial velocity permitting to glutamate to move into the synaptic cleft by Brownian motion [15].

During their Brownian motion (random walk) into the synaptic cleft, some of the neurotransmitter molecules can bind to the postsynaptic receptors. Movements of the neurotransmitter in the cleft is limited only by the synaptic structures. Diffusion can occur also laterally out of the synaptic space and the molecules of glutamate diffusion out of the synapse (spill over) are kept by the surrounding glial cell. The free motion is not only limited by the pre and postsynaptic surface. Other obstacles are the receptors itself, because they protrude from the PSD of almost $7 \mathrm{~nm}$, and the fibrils (14nm diameter) which are located around the PSD and AZ areas and connect the pre and postsynaptic membrane [16-17]. All these structure act as reflecting boundaries but their interaction with molecules of glutamate can contribute to shape the time course of synaptic response [17]. When at least two molecules of glutamate bound a postsynaptic receptor, it can open the gate permitting the flow of a depolarizing current called Excitatory Post Synaptic Current (EPSC). The EPSC produces a postsynaptic variation of the membrane potential called Excitatory Post Synaptic Potential (EPSP). If we assume that a presynaptic spike is the elementary bit of information ("a single letter") in a sequence of spikes representing a byte of information ("a whole word") then e single EPSP represents the single bit of information translated into the synaptic code [18].

However, what will be stressed in the present paper is that the characteristics of the produced EPSPs following a sequence of spikes are such that the code is not transmitted as it is. The EPSC, for example, also if we consider a single synapse, has an amplitude ranging from $4 \mathrm{pA}$ to $100 \mathrm{pA}$ [19-22] and the probability that a presynaptic spike reaching a glutamatergic terminal produces an EPSC (EPSP) is less than 1 . This variability not only makes difficult the interpretation of the synaptic code but make difficult also the understanding of how the postsynaptic code is formed. The EPSC variability is then a serious challenge for the understanding of the neurons and brain functioning. By following the scheme of Figure 1 , in the next sections the most important origins of this variability will shortly be described.

\section{Presynaptic Mechanisms of the EPSP/EPSC Variability}

As shown in the scheme of Figure 1, the Probability $\left(\mathrm{P}_{\mathrm{r}}\right)$ that a vesicle releases its glutamate following a presynaptic spike is a first important factor for defining the correspondence between the presynaptic neural code and the synaptic one. Some activity dependent modifications of the synaptic efficacy, like for example Short Term Potentiation (STP), Long Term Potentiation (LTP) and
Long Term Depression (LTD), which are the basic mechanisms involved in memory and learning, have been attributed by some authors to variations of $\mathrm{P}_{\mathrm{r}}$ (among many others, see for example) [23]. This point is strongly related to the point 3 of the scheme because the release probability depends on the interaction between the $\mathrm{Ca}^{2+}$ concentration at the presynaptic terminal and the SNARE complex [24]. The SNARE complex is a proteinic formation connected to the docking systems of the vesicles and $\mathrm{Ca}^{2+}$ transiently changes following a spike. Different configurations of the SNARE complex imply different interaction with $\mathrm{Ca}^{2+}$ which lead to different values $\operatorname{Pr}[24]$.

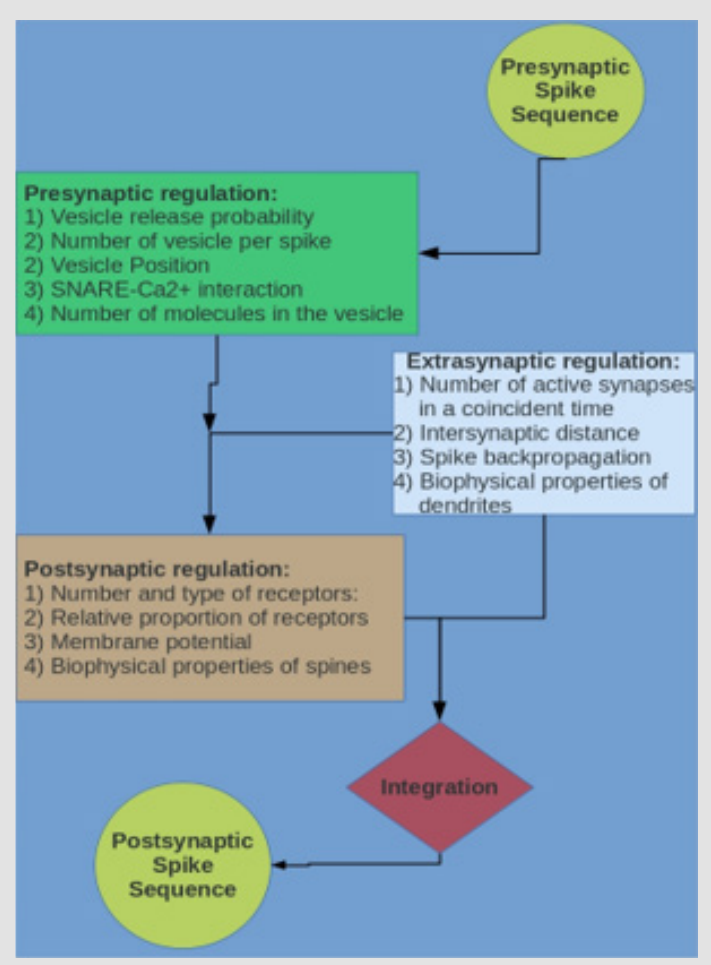

Figure 1: Schematic representation of the flow of information in the glutamatergic synapse.

Usually, each presynaptic spike has a $\mathrm{P}_{\mathrm{r}}$ less than 1 (range $0.2 \sim 0.91$ ) [25-26] and this rise a new important problem: whatever will be the value of $P_{r}$, being less than 1 means that there is a probability that not all the information encoded in the presynaptic spike sequence is transmitted [23] (i.e., there is not always a direct correspondence between the spike sequence and the synaptic EPSP sequences). In some way, the interaction between the $\mathrm{Ca}^{2+}$ and the SNARE complex "decide", depending on the state of excitation of the presynaptic neuron and on the local concentration of $\mathrm{Ca}^{2+}$, which bit of information should be transferred and which not. Some authors consider that, although $\mathrm{P}_{\mathrm{r}}$ is less than 1 , several docked vesicles can have the associated SNARE complex ready for the release (primed) and that, consequently, more than one vesicle can be released for a single spike (see for example) [25]. The multi-vesicular release for glutamatergic synapses is, however, a matter of debate mostly for its role in LTP production because an excess of neurotransmitter 
produced by a multivesicular release for a single spike can cause saturation and desensitization of the postsynaptic receptors reducing the clearance time of the glutamate from the synaptic cleft.

This, depending on the inter spike interval (ISI), in some cases would nullify the action of the spikes following the first one in a sequence because the saturated (or inactivated) receptors would not give contribute to the EPSP following the first one. Two other important factors influence from the presynaptic side the amplitude of the postsynaptic response. One is the position (eccentricity) of the releasing vesicle with respect to the central axis of the synapse and the other is the number of molecules of neurotransmitter into the releasing vesicle. The concentration of glutamate in the vesicle, in fact, ranges $60-210 \mathrm{mM}$ with an average of $140 \mathrm{mM}$ [26] and the number of molecules, depending also on the variability of the size of the vesicle, ranges from 500 up to more than 3000 for vesicle. Clearly a different number of molecules produces a different shaping of the concentration time course in the synaptic cleft which, in turn, produces different probability which a molecule meets a binding site of the receptors [26-28]. The correlation between a given spike and the number of molecules into the vesicle has been attributed to stochastic process [27-29].

The position of the vesicle with respect to the central axes of the synapse also play an important role [24-32]. As for the number of molecules in the vesicle, also the position is considered a mechanism of modulation of the postsynaptic response of stochastic origin [18,25,28-33].

\section{Postsynaptic and Extrasynaptic Mechanisms of The EPSP/EPSC Variability}

Although in Figure 1 it is shown a different area for postsynaptic and extrasynaptic mechanisms of regulation of information transfer in neurons, this distinction is only to show that the postsynaptic are intrinsic to the synaptic structure while the for extrasynaptic are external but noth of them act at the postsynaptic side. Probably a better division would be in intrinsic and extrinsic postsynaptic mechanisms of regulation of the EPSP (EPSC) amplitude. Hopefully this difference will result clear in the following. To understand these mechanisms firstly we need understand how the postsynaptic response is generated. The binding of at least two molecules of glutamate to a receptor has two different effect depending on the receptor type. While for AMPA receptors this is a necessary and enough condition to prode a depolarizing current, for the NMDA receptor it is a necessary but not sufficient condition. The gating of the NMDA receptors, in fact, are blocked by $\mathrm{Mg}^{2+}$ and the unblocking depends on the level of depolarization of the postsynaptic membrane which is a second necessary condition for these receptors to open and produce a postsynaptic current [34-35]. This property is considered the most important for the formation of the postsynaptic LTP, memory and learning because only levels of excitation sufficiently high ("information important to remember") produce their activation.
The most commonly accepted mechanisms for the production of the postsynaptic LTP involve the activation of NMDA receptors with a consequent $\mathrm{Ca}^{2+}$ influx which activates intracellular mechanisms which produces a variation of the number of AMPA and an increased postsynaptic response (among many other see [37] and bibliography therein). Several factors, some intrinsic to the synapse and some of extrasynaptic origin can induce activation of the NMDA response. The larger part of the post and extrasynaptic factors influence the dynamics of the postsynaptic response modulating the membrane level of depolarization. This is easily understood if we see the equation governing the postsynaptic potential at the spine level. In the simplest way, we can write

$$
\begin{gathered}
I(t)=G_{\text {syn }}(t)\left(V_{m}-V_{\text {rev }}\right) \\
V_{m}(t)=I(t) R_{i}
\end{gathered}
$$

Where I is the current produced by the receptors opening (EPSC), $\mathrm{G}_{\text {syn }}$ is the total conductance (AMPA+ NMDA conductance) and $V_{\text {rev }}$ is the synaptic reverse potential (a constant which for glutamatergic synapse is close to $0 \mathrm{mV}$ ), $\mathrm{V}_{\mathrm{m}}$ is the membrane potential and $\mathrm{R}_{\mathrm{i}}$ is the synaptic input resistance. The total conductance depends on the number and type of receptors which are opened at the time t. About the type of receptors, we must note that both AMPA and NMDA are tetrameric proteins composed of dimer of dimers. The different dimer composition for each type of receptor furnish different levels of the maximal single channel conductance that the receptors can express ([38-38] and bibliography therein).

The membrane potential $\left(\mathrm{V}_{\mathrm{m}}\right)$ depends on several factors. The input resistance $\mathrm{R}_{\mathrm{i}}$ is particularly high since the spine represents a separate electrical compartment with a high input impedance $[10,38-42]$. This point is not negligible because it means that a small current can produce a high value of $V_{m}$. [38-42]. From the above equations if follows that the synaptic response, for a given number of active receptors depends on the value of $V_{m}$. Any postsynaptic activity which modulates or change $V_{m}$ is automatically a source of postsynaptic response variability. For example, the fast activation of AMPA receptors following the release of a single vesicle, thanks to the high input resistance of the PSD, can depolarize the PSD sufficiently to activate few NMDA receptors even if the starting value of $V_{m}$ is close to the resing potential (-65 mV) [38-39]. In the real neuron, where many synapses can be active at the same time, a very strong contribution to the variability of $\mathrm{V}_{\mathrm{m}}$, and consequently of the EPSP, is given by the activity of pool of synapses active in the same time window (coincidence) positioned at a distance such to modify the membrane potential under the synapse [40-42].

The degree of modulatation by these the external synaptic pool depends on the number of active synapses and on their firing frequency [40-41]. For each size of the pool (number of active synapse) and for each mean firing frequency of the synapses of the pool, it is possible to identify a band of voltage, considered as a subset of values of membrane potential at which the synaptic 
response can occur [40-42]. The amplitude of each response for a given band of potential depends on the level of $V_{m}$ at the time of occurrence [41-42] of the synaptic vesicle release. In the dendritic tree, positioned on the dendritic shaft, there are also inhibitory $\mathrm{GABA}_{\mathrm{A}}$ synapses. Their activity contributes to define the limits of modularity imposed by the synaptic active pool [42].

Notably, all the regulatory effects inducing membrane depolarization favor the increase of the depolarization which lead to the $\mathrm{Mg}^{2+}$ removal and to the activation of the NMDA receptors. Similarly, the repolarizing contribution given by the GABA $A_{A}$ inhibitory synapse contribute less to the open of the NMDA but significantly increases the amplitude of the EPSP. The interplay of these mechanisms plays a very important role not only in shaping the EPSP but also in regulating the LTP and LTD [42] indicating that these phenomena, also if recorded in single synapses, probably depend mostly on the cooperativity among many synapses than on the single synapse activity [42]. The number of postsynaptic receptors change as a function of the synaptic activity and consequently changes the response amplitude. However, as seen in the previous paragraph, this is also depending on the level of the membrane potential. The number of AMPA in fact, vary as function of the activity from 0 (synapses AMPA silent) to more than 70 thanks to the activation of the NMDA and the consequent $\mathrm{Ca}^{2+}$ influx that, in turn, depends on the membrane voltage.

\section{Discussion}

The problem of how the synapses transfer information from one neuron to another is still far to be resolved. This is especially true for the glutamatergic synapse where the probability of release a vesicle by a single presynaptic spike is less than one. This is the main reason why it is not easy to define a synaptic code and its correspondence with the (presynaptic) neural code. Evidently, while the neural code is embedded in spike sequence, the synaptic code does not follow this rule. Moreover, while spikes are stereotyped events obeying to the "all or none" law (all of the same shape and amplitude), the shape and amplitude of the corresponding EPSP is variable and regulated by many pre, post and extrasynaptic factors. This not only makes very difficult to identify a synaptic code corresponding to the presynaptic one but make difficult also the understanding of the neural code arising on the postsynaptic neuron because it is generated by the integration of many synaptic inputs. It is extremely relevant the effect, at the postsynaptic side, of the other synapses active in a coincident time. Imagine, for example, that a group of synapses fires before another group, the action of the first group will depolarize the postsynaptic membrane and, consequently, the EPSPs produced by a second group will have a lower amplitude (see equation above).

This means that an excited area of the brain inputting on a neuron can reduce the effect of another area the input of which arrives on the neuron with some delay. In the case the depolarization produced by the first input rise the membrane potential up to the synaptic reversal potential the current produced by the synapses of the second arriving inputs will be zero and the information they carry can be nullified [43]. From these consideration it is clear that the study of the single synapse and of the way it transfer information among neurons is fundamental to have a better understanding not only of how the neural code is transmitted but also of the way the brain give priority to the information to process in order to produce the correct outputs.

\section{References}

1. Gulyás AI, Megías M, Emri Z, Freund TF (1999) Total number and ratio of excitatory and inhibitory synapses converging onto single interneurons of different types in the CA1 area of the rat hippocampus. Journal Neuroscience 199(22): 10082-10097.

2. Megías M, Emri Z, Freund TF Gulyás AI (2001) Total number and distribution of inhibitory and excitatory synapses on hippocampal CA1 pyramidal cells. Neuroscience 102(3): 527-540.

3. Gardoni F Di Luca M (2015) Targeting glutamatergic synapses in Parkinson's disease. Current Opinion in Pharmacology 20: 24-28.

4. Sheng M, Sabatini BL, Südhof TC (2012) Synapses and Alzheimer's Disease. Cold Spring Harb Perspect Biol 4:(5)1-18.

5. Rojas DC (2014) The role of glutamate and its receptors in autism and the use of glutamate receptor antagonists in treatment. J Neural Transm 121(8): 891-905.

6. Di Maio V, Santillo S, Ventriglia F (2018) Multisynaptic cooperation shapes single glutamatergic synapse response. Brain Research 1697: 93-104.

7. Tsay D, Yuste R (2004) On the electrical function of dendritic spines. Trends in Neurosciences 27(2): 77-83.

8. Araya R, Jiang J, Eisenthal, KB, Yuste R (2006) The spine neck filters membrane potentials. PNAS 103(47): 17961-17966.

9. Arellano JI, Benavides Piccione R, De Felipe J, Yuste, R (2007) Ultrastructure of dendritic spines: Correlation between synaptic and spine morphologies. Frontiers in Neuroscience 1: 132-142.

10. Yuste R (2013) Electrical Compartmentalization in Dendritic Spines. Annual Review of Neuroscience 36: 429-449.

11. Merriam EB, Millette M, Lumbard DC, Saengsawang W, Fothergill T, et al. (2013) Synaptic regulation of microtubule dynamics in dendritic spines by calcium, F-Actin, and drebrin. Journal Neuroscience 33(42): 1647116482.

12. Kwon T, Sakamoto M, Peterka DS, Yuste R (2017) Attenuation of Synaptic Potentials in Dendritic Spines. Cell Rep 20(5): 1100-1110.

13. Di Maio V, Santillo S, Sorgente A, Vanacore P, Ventriglia F (2018) Influence of active synaptic pools on the single synaptic event. Cognitive Neurodynamics 12(4): 391-402.

14. Di Maio V, Santillo S, Ventriglia F (2018) Multisynaptic cooperation shapes single glutamatergic synapse response. Brain Research 1697: 93-104.

15. Ventriglia F, Di Maio V (2003) Synaptic fusion pore structure and AMPA receptors activation according to Brownian simulation of glutamate diffusion. Biological Cybernetics 88(3): 201-209.

16.Zuber B, Nikonenko I, Klauser P, Muller D, Dobochet J (2005) The mammalian central nervous synaptic cleft contains a high density of periodically organized complexes. PNAS 102(52): 19192-19197.

17. Ventriglia F (2011) Effect of filaments within the synaptic cleft on the response of excitatory synapses simulated by computer experiments. Biosystems 104(1): 14-22.

18. Di Maio V Santillo S (2020) Information Processing and Synaptic Transmission In: Advances in Neural Signal Processing. Intech Open. 
19. Forti L, Bossi M, Bergamaschi A, Villa A, Malgaroli A (1997) Loose path recording of single quanta at individual hippocampal synapses. Nature 388(6645): 874-878.

20. Auger C, Martin A (2000) Quantal currents at single-site central synapse. Journal of Physiology 526(1): 3-11.

21. Hanse E, Gustafsson B (2001) Quantal variability at glutamatergic synapses in area CA1 of the rat neonatal hippocampus. Journal of Physiology 531(2): 467-480.

22. Di Maio V, Ventriglia F, Santillo S (2017) Stochastic, structural and functional factors influencing AMPA and NMDA synaptic response variability: a review. Neuronal Signaling 1(3): 1-11.

23. Kokaia M (2000) Long-term potentiation of single subicular neurons in mice. Hippocampus 10(1): 684-692.

24. Guerrier C, Holcman D (2018) The First 100 nm Inside the Pre-synaptic Terminal Where Calcium Diffusion Triggers Vesicular Release. Frontiers in Synaptic Neuroscience 10:23.

25. Dobrunz LE, Stevens CF (1997) Heterogeneity of release probability, facilitation, and depletion at central synapses. Neuron 18(6): 995-1008.

26. Park H, Li Y, Tsien RW (2012) Influence of Synaptic Vesicle Position on Release Probability and Exocytotic Fusion Model. Science 335(6074): 1362-1366

27. A Loebel, Gilad Silberberg, Daniela Helbig, Henry Markram, Misha Tsodyks, et al. (2009) Multiquantal release underlies the distribution of synaptic efficacies in the neocortex Frontiers in Computational Neuroscience

28. Clements JD (1996) Transmitter timecourse in the synaptic cleft: its role in central synaptic function. Trends in Neurosciences, 19(50: 163-171.

29. Ventriglia F, Di Maio V (2000) A Brownian simulation model of glutamate synaptic diffusion in the femtosecond time scale. Biological Cybernetics 83(2): 93-109.

30. Ventriglia F, Di Maio V (2002) Stochastic fluctuation of the synaptic function. Biosystems 67(1-3): 287-294.

31. Ventriglia F Di Maio V (2003) Stochastic fluctuation of the quantal EPSC amplitude in computer simulated excitatory synapses of hippocampus. Biosystems 71(1-2): 195-204.

\section{ISSN: 2574-1241}

DOI: 10.26717/BJSTR.2019.23.003947

Vito Di Maio. Biomed J Sci \& Tech Res

(C) This work is licensed under Creative

Submission Link: https://biomedres.us/submit-manuscript.php
32. Ventriglia F, Di Maio V (2003) Stochastic fluctuation of the quantal EPSC amplitude in computer simulated excitatory synapses of hippocampus. Biosystems 71(1-2): 195-204.

33. Di Maio V, Ventriglia F, Santillo S (2017) Stochastic, structural and functional factors influencing AMPA and NMDA synaptic response variability: a review. Neuronal Signaling 1(2): 1-11.

34.Jahr CE, Stevens CF (1990) Voltage Dependence of NMDA-Activated macroscopic conductances predicted by single-channel kinetics. Journal Neuroscience 10(9): 3178-3182.

35. Vargas Caballero M, Robinson HP (2004) Fast and slow voltagedependent dynamics of magnesium block in the NMDA receptor: The asymmetric trapping block model. Journal of Neuroscience 24(27): 6171-6180.

36. Ventriglia F, Di Maio V (2013) Effects of AMPARs trafficking and glutamate-receptor binding probability on stochastic variability of EPSC. Biosystems 112(3): 298-304.

37. Sjöströ PJ, Turrigiano G, Nelson SB (2001) Rate, Timing, and Cooperativity Jointly Determine Cortical Synaptic Plasticity. Neuron 32(6): 1149-1164.

38. Di Maio V, Ventriglia F, Santillo S (2016) A model of cooperative effect of AMPA and NMDA receptors in glutamatergic synapses. Cognitive Neurodynamics 10(4): 315-325.

39. Di Maio V, Ventriglia F, Santillo S (2016) AMPA/NMDA cooperativity and integration during a single synaptic event. Journal of Computational Neuroscience 41(2): 127-142.

40. Di Maio V, Santillo S, Sorgente A, Vanacore P, Ventriglia F (2018) Influence of active synaptic pools on the single synaptic event. Cognitive Neurodynamics 12(4): 391-402.

41. Di Maio V, Santillo S, Ventriglia F (2018) Multisynaptic cooperation shapes single glutamatergic synapse response. Brain Research 1697: 93-104.

42. Di Maio V, Santillo S, Ventriglia F (2020) Dendritic modulation of the single synaptic event. Cognitive Neurodynamics (in press).

43. Di Maio V (2008) Regulation of information passing by synaptic transmission: a short review. Brain Research, 1225: 26-38.

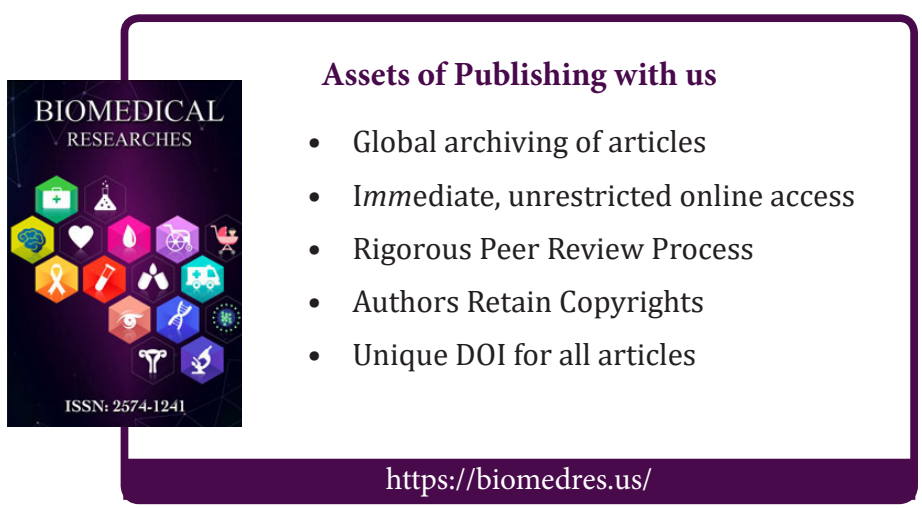

